\title{
Early clinical predictive factors during coma recovery
}

\author{
R. Formisano ${ }^{1}$, U. Bivona ${ }^{1,2}$, F. Penta ${ }^{1}$, M. Giustini ${ }^{2}$, M. G. Buzzi ${ }^{1}$, P. Ciurli ${ }^{1}$, M. Matteis ${ }^{1}$, C. Barba ${ }^{1}$, \\ C. Della Vedova ${ }^{1}$, V. Vinicola ${ }^{1}$, and F. Taggi ${ }^{2}$ \\ ${ }^{1}$ I.R.C.C.S. Rehabilitation Hospital, Santa Lucia Foundation, Rome, Italy \\ ${ }^{2}$ National Institute of Health, Rome, Italy
}

\section{Summary}

In severe brain injury patients few studies have examined the role of early clinical factors emerging before recovery of consciousness. Patients suffering from vegetative state and minimally conscious state in fact may need variable periods of time for recovery of the ability to follow commands. In a previous study we retrospectively examined a population of very severe traumatic brain injury patients with coma duration of at least 15 days (prolonged coma), and we found, as significant predictive factors for the final outcome, the time interval from brain injury to the recovery of the following clinical variables: optical fixation, spontaneous motor activity and first safe oral feeding. Psychomotor agitation and bulimia during coma recovery were also favourable prognostic factors for the final outcome. In a further study, also as for the neuropsychological recovery, the clinical variable with the best significant predictive value was the interval from head trauma to the recovery of safe oral feeding.

In the present study the presence of psychomotor agitation diagnosed by means of LCF (score $4=$ confused-agitated) at the admission time in rehabilitation predicted a statistically significant better outcome at the discharge time in comparison with patients without agitation.

Keywords: Traumatic brain injury; prolonged coma; outcome; predictive factors; psychomotor agitation.

\section{Introduction}

Survivors from severe traumatic brain injury (TBI) often suffer from prolonged disturbances of consciousness, such as coma (lasting from few hours to some days), prolonged coma (lasting at least 15 days), minimally conscious state [12] and/or vegetative state [16]. These conditions may be followed by different clinical outcomes, with recovery process lasting variable periods of time (from few days to several months). Age, severity and duration of coma, duration of posttraumatic amnesia, site and extent of cerebral lesions and association with polytrauma and hypoxia have been considered as the main prognostic factors to pre- dict the outcome of severe TBI patients $[1,5,10,17-$ 19]. Unfortunately, useful studies looking at a variety of clinical features observed in the early phases of coma recovery are scant. The majority of the studies investigating the clinical factors predicting the final outcome of severe brain injury patients regard the acute phase. In fact, coma severity, measured by means of Glasgow Coma Scale (GCS) [22], is correlated with the final outcome, assessed by the Glasgow Outcome Scale (GOS) [15], although it is well known that the GOS score may still change within the first year from brain injury. Coma duration is also a significant predictive factor for final outcome of severe brain injury patients, but few studies have examined the role of early clinical factors emerging before the complete recovery of consciousness. Patients suffering from vegetative state and minimally conscious state in fact may need variable periods of time for recovery of the constant ability to follow commands. The duration of post-traumatic amnesia, which is a significant prognostic factor for final outcome of TBI patients, is very difficult to be measured in minimally conscious state or patients suffering from persistent memory disturbances.

In a previous study we retrospectively examined a population of severe traumatic brain injury patients with coma duration of at least 15 days (prolonged coma), in order to evaluate some possible clinical predictive factors for the final outcome. A statistically significant correlation with disability scales such as GOS and Barthel Index (BI) at 1 year follow up was found for the time interval from brain injury to the recovery of the following clinical variables: optical fixation, ability to follow commands, spontaneous 
Table 1. Post-Coma Scale (1-60)

Pupils

$4=$ bilateral prompt reaction

$3=$ monolateral prompt reaction

$2=$ torpid reaction

$1=$ mild signs of reaction

$0=$ no reaction - fixed mydriasis

Reaction to auditory stimulation

$4=$ obeys to command

$3=$ looks toward the stimulation

$2=$ blinks in response to the stimulation

$1=$ does not look to the stimulation

$0=$ no reaction

Reaction to painful stimulation

$4=$ finalized motor response

$3=$ localizes, but not ward off pain

$2=$ decorticated reaction

$1=$ decerebrated reaction

$0=$ no reaction

Eyelid and ocular bulbi position

$4=$ eyes open spontaneously

$3=$ eyes open to stimulation

$2=$ eyes open to painful stimulation

$1=$ fixed divergence of the bulbi

$0=$ eyes closed

Motility of the eyes

$4=$ ocular pursuing

$3=$ blinks to menace

$2=$ absent oculocephalic response

$1=$ present oculocephalic response

$0=$ pendular movements of the eyes

Oral movements

$4=$ normal (follows commands)

$3=$ bite tendency and oral exploration

$2=$ trismus, jaw contracture

$1=$ primitive oral automatism ( jawning, sucking, bruxism, etc.)

$0=$ no oral movements

Spontaneous motility and posture

$4=$ normal

$3=$ spontaneous motor activity

$2=$ decortication

$1=$ decerebration

$0=$ flaccidity

Speech

$4=$ normal

$3=$ aphonia or dysarthria

$2=$ confused and stereotyped words/confabulations

$1=$ incomprehensible sounds

$0=$ no speech

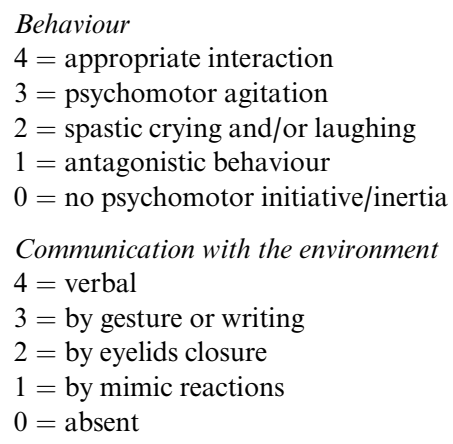

Duration of consciousness disturbance (at the evaluation time)

$4=$ less than 1 month

$3=$ less than 3 months

$2=$ less than 6 months

$1=$ less than 1 year

$0=$ longer than 1 year

Breathing

$4=$ normal

$3=$ tachypnoea or stertorous

$2=$ with pauses/periodic breathing

$1=$ intubation/tracheostomy

$0=$ assisted ventilation

Feeding

$4=$ normal

$3=$ dysphagia

2 = food refusal (incostant feeding by mouth)

$1=$ stomach tube/gastrostomy $(\mathrm{PEG})$

$0=$ parenteral nutrition

Sphincters' control

$4=$ normal

$3=$ sporadic incontinence and/or retention

$2=$ urine condom $/$ napkin

$1=$ vesicostomy/intermittent catheterization

$0=$ no control (permanent urine catheter and fecal incontinence)

Cutaneous trophysm

$4=$ normal

$3=$ small non infected bedsore

$2=$ wide non infected bedsore

$1=$ infected bedsore or multiple bedsores

$0=$ multiple wide and/or infected bedsores motor activity and first safe oral feeding. Psychomotor agitation and bulimia during coma recovery were also favourable prognostic factors for the final outcome [8].

In a further study we evaluated the possible role of some clinical factors in predicting cognitive outcome in a group of TBI patients, with GCS lower than 8 and prolonged coma. The clinical variables evaluated in correlation with the neuropsychological outcome were the following: age, duration of unconsciousness, duration of post-traumatic amnesia, interval from head trauma to neuropsychological evaluation, interval from head trauma to recovery of oral feeding, and 
finally interval from head trauma to first verbal communication. The clinical variable with a significant predictive value on most neuropsychological scores was the interval from head trauma to the recovery of safe oral feeding. Length of unconsciousness and the time interval between head trauma and both first verbal communication and oral feeding were negatively correlated with the global measure of disability as expressed by the GOS score in the expanded version [9].

Clinical observations have suggested that traumatic brain injury patients go through a stage of agitation and restlessness as a natural part of recovery process $[2-4,21]$. Moreover, psychomotor agitation and restlessness are reported as favourable prognostic features of recovery from severe brain injury $[2,21]$.

Aim of this study was to confirm the possible prognostic role of some clinical factors emerging during recovery of consciousness in severe brain injury patients with prolonged coma, such as the presence of psychomotor agitation, for predicting the final outcome.

\section{Materials and methods}

We enrolled 150 severe brain injury patients, consecutively admitted to the Rehabilitation Hospital Santa Lucia in Rome, from October 2001 to October 2003, as in- or out-patients. The collection of data was performed to join in a multicentric Italian study on severe acquired cerebral lesions (GISCAR), involving 66 rehabilitation centres for severe brain injury.

At the admission to our Rehabilitation Hospital $\left(\mathrm{t}_{0}\right)$ and at discharge $\left(t_{1}\right)$ the following disability scales were administered to all patients:

- Levels of Cognitive Functioning (LCF) [14];

- Disability Rating Scale (DRS) [20];

- Glasgow Outcome Scale (GOS).

In order to obtain a single "improvement index" (I.I.), LCF, DRS and GOS were summed up and normalized, to make the interval between scores homogeneous for the 3 scales. In particular, since DRS has a total range score of $30, \mathrm{LCF}$ of 8 and GOS of 5, LCF was multiplied for $3.75(8 \times 3.75=30)$, whereas GOS was multiplied for $6(5 \times 6=30)$. I.I. was finally obtained by the difference of the normalized sums of the 3 scales at $t_{0}$ and $t_{1}$ times.

In a minority of patients (30 patients) Post-Coma Scale (PCS) [7] (Attached) was administered at admission to our Rehabilitation Hospital by two blind examiners, to correlate PCS with DRS and to evaluate the inter-rater reliability of PCS. Correlation between the 2 examiners (PCS1 and PCS2) and the 2 disability rating scales was examined by means of Spearman R test. P level was set up at 0.05 .

Finally, age, etiology of coma, interval from coma to admission in rehabilitation and the presence of psychomotor agitation during coma recovery were investigated in correlation to the outcome at discharge from rehabilitation.

Statistical analysis of the data was performed by means of non parametric tests such as Kruskal-Wallis, Fisher exact test and by logistic analysis.

\section{Results}

Among the 150 severe brain injury patients admitted as in-patients, day hospital or out-patients, 80 in-patients at the first rehabilitation admission were examined.

The 80 patients $(57 \mathrm{M}, 23 \mathrm{~F})$ had a mean age of 38 years (range: from 18 to 78 ) and a mean coma duration of 29 days (range: $2-180$ days). The etiology of brain injury was: traumatic brain injury (TBI) $(\mathrm{N}=51$ patients; $63.8 \%)$; hypoxic coma ( $\mathrm{N}=8$ patients; $10.0 \%)$; hemorrhagic stroke, including non traumatic subarachnoid hemorrhage ( $\mathrm{N}=17$ patients; $21.2 \%$ ); ischaemic stroke $(\mathrm{N}=2$ patients; $2.5 \%)$; cerebral infections meningo-encephalitis ( $\mathrm{N}=2$ patients; $2.5 \%$ ). The mean length of stay, including the whole period of rehabilitation (as in-patient, day hospital, out-patient) was of 161 days (range: 23-519 days).

Among TBI patients $(\mathrm{N}=51)$ only 5 patients $(9.8 \%)$ were older than 50 years and none of them had a significant improvement, i.e. improvement index (I.I.) higher than the median score. Conversely, among patients younger than 50 years $(90.2 \%)$, in 28 patients $(60.9 \%)$ the improvement was statistically significant (Table 2). Patients younger than 40 years had a probability to improve higher (about six times) than patients older than 40 years, with a trend to a statistically significance $(\mathrm{p}<0.06)$.

As for the etiology, the improvement index (I.I.) of traumatic patients was higher than non traumatic cases (15.7 vs 11.2$)$, with a trend to statistical significance $(\mathrm{p}<0.09)$ (Table 3$)$.

Table 2. Improvement index as related to patients' age

\begin{tabular}{llcc}
\hline Age & \multicolumn{2}{l}{ Improvement index } & Total $(\%)$ \\
\cline { 2 - 3 } & Improvement & No improvement & \\
\hline$<50$ years & $28(60.9 \%)$ & 18 & $46(90.2 \%)$ \\
$>50$ years & 0 & 5 & $5(9.8 \%)$ \\
Total & 28 & 23 & 51 \\
\hline
\end{tabular}

Table 3. Comparison between improvement index from traumatic (TBI) and non traumatic brain-injured (non-TBI) patients

\begin{tabular}{llllrr}
\hline & $\begin{array}{l}\text { Patients } \\
\text { observed }\end{array}$ & Total I.I. & Mean & Variance & Std Dev \\
\hline TBI & 51 & 801.50 & 15.71 & 139.71 & 11.82 \\
Non-TBI & 29 & 326.25 & 11.25 & 79.97 & 8.94 \\
\hline
\end{tabular}

$\mathrm{p}<0.09-$ Fisher exact test 0.0868 .

$\mathrm{p}<0.02-$ Fisher exact test 0.014 . 
Table 4. Comparison between Improvement Index in TBI patients with LCF score $=4$ (confused-agitated) and TBI patients with LCF scores different from 4 (not agitated)

\begin{tabular}{llll}
\hline & \multicolumn{2}{l}{ Improvement index } & \multirow{2}{*}{ Total } \\
\cline { 2 - 3 } & Improvement & No improvement & \\
\hline LCF $=4$ & $15(71.4 \%)$ & 6 & $21(41.2 \%)$ \\
LCF non 4 & $13(43.3 \%)$ & 17 & $30(58.8 \%)$ \\
Total & 28 & 23 & 51 \\
\hline
\end{tabular}

$\mathrm{p}<0.05-$ Fisher exact test 0.043

$L C F$ Level of Cognitive Functioning.

Table 5. Improvement Index as related to time interval between TBI and admission at rehabilitation

\begin{tabular}{llll}
\hline Interval & \multicolumn{2}{l}{ Improvement index } & \multirow{2}{*}{ Total (\%) } \\
\cline { 2 - 3 } TBI/rehabilitation & Improvement & No improvement & \\
\hline$<90$ days & $22(68.8 \%)$ & $10(31.3 \%)$ & $32(62.7 \%)$ \\
$>90$ days & $6(31.3 \%)$ & $13(68.4 \%)$ & $19(37.3 \%)$ \\
Total & 28 & 23 & 51 \\
\hline
\end{tabular}

$\mathrm{P}<.001-$ Fisher exact test 0.0107.

Among the 51 patients, $21(41.2 \%)$ had an LCF score equal to 4 (confused-agitated) at admission in rehabilitation and among them a statistically significant higher percentage of patients showed an improvement index (I.I.) higher than the median score (equal to 13$)(\mathrm{p}<0.05)$.

Therefore the presence of psychomotor agitation diagnosed by means of LCF at the admission time $\left(\mathrm{t}_{0}\right)$ predicted a statistically significant better outcome at the discharge time $\left(\mathrm{t}_{1}\right)$ in comparison with patients without agitation (Table 4).

As for interval from brain injury and admission in rehabilitation, the beginning of rehabilitation within 90 days significantly increased the improvement probability of about 5.7 times $(\mathrm{p}<0.02)$ in comparison with patients admitted in rehabilitation later than 90 days after brain injury (Table 5).

This result was also confirmed by a logistic analysis including the different clinical factors, i.e. age, interval from brain injury to admission in rehabilitation and coma duration.

Finally, PCS, as a measure of global disability, including also the interval from coma to the evaluation time and the presence of bed rest syndrome, showed a good inter-rater reliability and a statistically significant correlation with the DRS score (Table 6).
Table 6. Correlation between two disability rating scales

\begin{tabular}{lcc}
\hline- & Spearman R & p-level \\
\hline PCS 1st-PCS 2st & 0.96 & 0.001 \\
DRS-PCS 1st & -0.88 & 0.001 \\
DRS-PCS 2st & -0.92 & 0.001 \\
\hline
\end{tabular}

1st First Examiner, 2st Second Examiner, PCS Post-Coma Scale, $D R S$ Disability Rating Scale.

\section{Discussion}

Vegetative state is usually defined as "the absence of any understandable response to external stimuli or inner need" [16]. However, such a definition raises the question of whether psychomotor agitation represents a lack of response to external stimuli or inner need. Restlessness and agitation in the first phase of coma recovery, or as behaviour emerging in severe brain injury patients with prolonged disturbances of consciousness, rarely leads to a significant functional interaction of the patient with the environment. Usually, in fact, the agitated patient does not follow commands, either for antagonistic behaviour or for extreme attention lability. Is therefore psychomotor agitation a minimally conscious state or a vegetative state? As a matter of fact, in spite of the lack to follow commands, restlessness and agitation may be interpreted as purposeful behaviour and might precede the recovery of consciousness.

In this preliminary study the presence of psychomotor agitation at the beginning of rehabilitation was a good predictive feature for recovery at the end of rehabilitation program.

Other behavioural disturbances have been previously reported in severe brain injury patients, such as Klùver-Bucy syndrome, i.e. presence of 3 or more of the following symptoms and signs: increased oral activity, hypersexuality, hypermetamorphosis (extreme attention lability), memory disorders, placidity, loss of people recognition, bulimia [13]. The syndrome has also been reported as a possible recovery phase and positive prognostic feature for a good recovery in patients with severe traumatic brain injury and prolonged disturbance of consciousness $[6,11]$.

In a previous study [8] we also demonstrated the positive predictive role of some clinical features emerging during recovery of consciousness in very severe brain injury with prolonged coma, for the final outcome, i.e. time interval from brain injury to the recovery of optical fixation, spontaneous motor activity 
and the first safe oral feeding. Moreover, as previously reported, in very severe brain injury patients with prolonged coma, also the neuropsychological outcome was best predicted by the time interval from brain injury to the recovery of the first safe oral feeding [9]. Psychomotor agitation and bulimia were also favourable prognostic factors for the final outcome [8].

As for spontaneous motor activity, it is a common clinical experience that patients with preserved spontaneous motor activity had better outcome than patients with pathological posturing of upper and lower limbs such as decerebrated or decorticated posture, which are commonly associated to vegetative or minimally conscious state.

In severe brain injury the most significant clinical prognostic factors are represented by severity of coma (GCS), coma duration and post-traumatic amnesia.

Very severe brain injury with prolonged coma (coma duration of at least 15 days) other clinical features emerging during coma recovery may be of interest, such as spontaneous motor activity, psychomotor agitation, hypersexuality (Klùver-Bucy) and the time interval from brain injury to recovery of safe oral feeding.

Finally, PCS, as a measure of global disability, including the interval from brain injury to the administration of the scale and need of intensive nursing care, may be an interesting evaluation tool for predicting final outcome in patients with prolonged disturbances of consciousness.

If our preliminary results will be confirmed in larger studies, spontaneous motor activity and psychomotor agitation might be favourable prognostic features for recovery of consciousness in vegetative and minimally conscious state.

\section{References}

1. Carlsson C, Von Essen C, Lofgren J (1968) Factors affecting the clinical course of patients with severe head injuries. Journal of Neurosurgery 29: 242-251

2. Corrigan JD, Mysiw WJ (1988) Agitation following traumatic head injury: equivocal evidence for a discrete stage of cognitive recovery. Arch Phys Med Rehabil 69(7): 487-492

3. Denny-Brown D. Cerebral concussion (1945) Physiol Rev 25: 296-325

4. Denny-Brown D. Intellectual deterioration resulting from head imjury (1945) Res Publ Ass Nerv Ment Dis (chapter 18) 24: 467 472

5. Espersen JO, Petersen OF (1982) Computerized tomography (CT) in patients with head injuries. Assessment of outcome based upon initial clinical findings and initial CT scans. Acta Neurochir (Wien) 65: 81-92

6. Formisano R, Saltuari L, Gerstenbrand F (1995) Presence of Klüver-Bucy syndrome as a positive prognostic feature for the remission of traumatic prolonged distirbances of cousciousness. Acta Neurol Scandinavica 91: 54-57

7. Formisano R, Vinicola V et al (1996) Assessment scale for patients with prolonged disturbances of consciousness (postcoma scale). Proceedings of the Fifth Conference of the International Association for the study of Traumatic Brain Injury and 20th Conference of the Australian Society for the Study of Brain Impairment, Melbourne, Australia, Nov 15-18

8. Formisano R, Voogt RD, Buzzi MG, Vinicola V, Penta F, Peppe A, Stanzione P (2004) Time interval of oral feeding recovery as a prognostic factor in severe traumatic brain injury. Brain Inj 18(1): 103-109

9. Formisano R, Carlesimo GA, Sabbadini M, Loasses A, Penta F, Vinicola V, Caltagirone C (2004) Clinical predictors and neuropsychological outcome in severe brain injury patients. Acta Neurochir (Wien) 146: 457-462

10. Frowein RA, Terhaag P, Firsching R (1989) Long lasting outcome after head injury: late results. Adv Neurosurg 17: 36-42

11. Gerstenbrand F, Poewe W, Aichner F, Saltuari L (1983) KlùverBucy syndrome in man: experiences with posttraumatic cases. Neurosci Biobehav Rev Fall 7(3): 413-417

12. Giacino JT, Ashwal S, Childs N, Cranford R, Jennett B, Katz DI, Kelly JP, Rosenberg JH, Whyte J, Zafonte RD, Zasler ND (2002) The minimally conscious state: definition and diagnostic criteria. Neurology 58(3): 349-353

13. Goscinski I, Kwiatkowski S, Polak J, Orlowiejska M, Partyk A (1997) The Klùver-Bucy syndrome. J Neurosurg Sci 41(3): 269 272

14. Hagen C, Malkmus D, Durham P (1979) Levels of cognitive functioning. Rehabilitation of the head injured adult. Comprehensive physical management: 8. Professional Staff Association of Rancho Los Amigos Hospital, Inc. Downey

15. Jennett B, Bond M (1975) Assessment of outcome after severe brain damage. Lancet 1(7905): 480-484

16. Jennett B, Plum F (1972) Persistent vegetative state after brain damage. RN 35(10): 1-4

17. Miller JD, Becker D, Ward J et al (1977) Significance of intracranial hypertension in severe head injury. J Neurosurg 47: $503-$ 516

18. Overgaard J, Christensen S, Hvid-Hassen O et al (1973) Prognosis after head injury based on early clinical examination. Lancet 2: 631-635

19. Pitts LH, Mc Intosh TK (1990) Dynamic changes after brain trauma. Handbook of clinical neurology, vol. 13 (57). Head injury. In: Braakman R (ed) Elsevier Svience Publ. B.V. Chapt. 4, p 65-100

20. Rappaport M, Hall KM, Hopkins K, Belleza T, Cope DN (1982) Disability rating scale for severe head trauma: coma to community. Arch Phys Med Rehabil 63: 132-141

21. Reyes RL, Bhattacharyya AK, Heller D (1981) Traumatic head Injury: Restlessness and agitation as prognosticators of physical and psychological improvement in patients. Arch Phys Med Rehabil 62: 20-23

22. Teasdale G, Jennett B (1974) Assessment of coma and impaired consciousness. A practical scale. Lancet 2(7872): 81-84

Correspondence: Rita Formisano, Fondazione Santa Lucia, Via Ardeatina 306, 00179 Rome, Italy. e-mail: r.formisano@ hsantalucia.it 